\title{
RNA-protein interactions in the yeast three-hybrid system: Affinity, sensitivity, and enhanced library screening
}

\author{
BRAD HOOK, DAVID BERNSTEIN, BEILIN ZHANG, ${ }^{1}$ and MARVIN WICKENS
}

Department of Biochemistry, University of Wisconsin, Madison, Wisconsin 53706, USA

\begin{abstract}
The yeast three-hybrid system has become a useful tool in analyzing RNA-protein interactions. An RNA sequence is tested in combination with an RNA-binding protein linked to a transcription activation domain (AD). A productive RNA-protein interaction activates a reporter gene in vivo. The system has been used to test candidate RNA-protein pairs, to isolate mutations in each interacting partner, and to identify proteins that bind a given RNA sequence. However, the relationship between reporter gene activation and in vitro affinity of an RNA-protein interaction has not been examined systematically. This limits interpretation of the data and complicates the development of new strategies. Here, we analyze several key parameters of the three-hybrid system, using as a model the interaction of a PUF protein, FBF-1, with a range of RNA targets. We compare activation of two reporter genes as a function of the in vitro affinity of the interaction. HIS3 and LacZ expression levels are directly related to affinity over a 10 -fold range of $\mathrm{Kd}$. Expression of the reporter genes also is directly related to the abundance of the activation domain fusion protein. We describe a new yeast strain, YBZ1, that simplifies screening of cDNA/AD libraries. This strain possesses a tandem, head-to-tail dimer of a high-affinity variant of MS2 coat protein, fused to a monomer of the LexA DNA-binding protein. We show that the use of this strain in cDNA library screens increases the number of genuine, sequencespecific positives detected, and at the same time reduces the background of false, RNA-independent positives.
\end{abstract}

Keywords: RNA-protein interactions; three-hybrid system

\section{INTRODUCTION}

RNA-protein interactions underlie diverse biological processes, from pattern formation to the replication of viruses. As a result, methods have been developed to analyze RNAprotein interactions using molecular genetics. For example, assays based on antitermination, retroviral replication, phage assembly, and phage display have been used to dissect specific interactions in detail, and to isolate RNAs and polypeptides with altered affinity or specificity (Harada et al. 1996, 2000; Jain and Belasco 1996, 2000; Laird-Offringa 1999; SenGupta et al. 1999; Celander et al. 2000; Cullen 2000; Kollmus and Hauser 2000; Landt et al. 2000; Paraskeva and Hentze 2000; Peled-Zehavi et al. 2000; Xie et al. 2004). A yeast three-hybrid system has enabled the identification of naturally occurring RNA and protein partners, and the dissection of higher-order RNA-protein complexes (Bernstein et al. 2002).

${ }^{1}$ Present address: Sangamo Biosciences, Richmond, CA 94804, USA. Reprint requests to: Marvin Wickens, Department of Biochemistry, 433 Babcock Drive, University of Wisconsin, Madison, WI 53706, USA; e-mail: wickens@biochem.wisc.edu; fax: (608) 262-9108.

Article published online ahead of print. Article and publication date are at http://www.rnajournal.org/cgi/doi/10.1261/rna.7202705.
In the yeast three-hybrid system, a chimeric protein containing both a DNA- and RNA-binding domain tethers an RNA to the promoter of a reporter gene (Fig. 1; SenGupta et al. 1996). Typically, this protein consists of a LexA/MS2 coat protein fusion. A hybrid RNA binds to the MS2 portion via tandem MS2-binding sites. The RNA also contains a sequence of interest, $\mathrm{X}$, which binds to an RNA-binding polypeptide, $\mathrm{Y}$. $\mathrm{Y}$ is linked to a transcription activation domain $(\mathrm{AD})$. When the requisite interactions occur, the reporter gene is activated. Typically, the interaction between RNA X and protein Y is monitored by assaying HIS3 and LacZ expression levels. Qualititatively, weak and strong interactions can be discriminated phenotypically using HIS3. This feature has been used to isolate mutations in the RNA or protein that alter affinity (for review, see Bernstein et al. 2002). However, a systematic examination of the relationship between in vitro affinity of an RNA-protein interaction and reporter gene activity has not been reported. This limits interpretation of the data, and hampers the design of new applications.

One widespread use of the system has been to identify proteins from cDNA libraries that bind to an RNA sequence of interest (Bernstein et al. 2002). Many factors contribute to the feasibility of such screens, some of which are beyond 


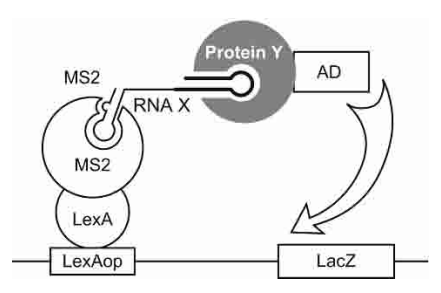

FIGURE 1. Three-hybrid system to detect and analyze RNA-protein interactions. Commonly used protein and RNA components are depicted. Both LacZ and HIS3 are present in strains L40coat and YBZ-1, and are placed under the control of LexA operators. The hybrid RNA contains two MS2-binding sites, and each of the reporters possesses multiple LexA operators.

the reach of the investigator: the abundance of the cognate cDNA in the library and the affinity of the interaction both are critical, for example. On the other hand, methods have been developed to help identify and eliminate proteins that bind to the LexA/MS2 fusion protein directly, without binding RNA (Park et al. 1999; Bernstein et al. 2002). Even with these improvements, this class of false positives can constitute a large fraction of the total number of transformants, and so make screening more laborious and problematic.

In this report, we focus on two key aspects of the yeast three-hybrid system to inform and enable its use. First, we examine the relationship between reporter gene activation and the in vitro affinity of the interaction, as well as the abundance of the protein partner. Second, we describe a new yeast strain that facilitates the detection of proteins in cDNA libraries that bind specifically to an RNA of interest.

\section{RESULTS}

\section{LacZ versus $H I S 3$ reporters}

In the yeast three-hybrid system, RNA-protein interactions are commonly monitored by measuring the level of expression of LacZ and HIS3 reporter genes. Both are under the control of the LexA operator. LacZ is monitored by measuring $\beta$-galactosidase activity, and HIS3 by growth phenotype. We first determined the relationship between the expression of these two reporter genes as a function of RNAprotein affinity.

For this purpose, we used Caenorhabditis elegans FBF-1 protein and six RNAs to which it binds. FBF-1 is a member of the PUF protein family (Wickens et al. 2001, 2002). The six RNAs analyzed here comprise two wild-type sites, the FBEa (Crittenden et al. 2002) and the PME (Zhang et al. 1997) and four point mutant derivatives of the FBEa site (D. Bernstein, B. Hook, and M. Wickens, in prep.). Together these encompass a 10 -fold range of $\mathrm{Kd}$, from 10 to $100 \mathrm{nM}$ (see below). The FBEa and PME RNA sequences used are found in the 3'-UTRs of the gld-1 and fem-3 mRNAs, respectively. In vivo, FBF binds the FBEa in gld-1 mRNA to control proliferation of germ-line stem cells (Crittenden et al. 2002, 2003) and the PME in fem-3 mRNA to control the switch from spermatogenesis to oogenesis in hermaphrodites (Kraemer et al. 1999).

The yeast strain, YBZ1, was transformed with plasmids carrying FBF-1 linked to an activation domain (FBF-1/AD) and one of six RNAs. (The YBZ1 strain is described in greater detail in Fig. 5, below.) The level of expression of HIS3 and LacZ genes was then determined for each combination. To analyze HIS3, we examined growth in the absence of histidine and presence of varying concentrations of 3-aminotriazole (3-AT). 3-AT is a competitive inhibitor of His $3 p$; cells containing more His3p survive at higher concentrations of 3-AT. To monitor LacZ expression, $\beta$-galactosidase activities were assayed by measuring the conversion of a lactose analog to a luminescent product using a simple protocol (see Materials and Methods).

HIS3 and $\beta$-galactosidase activity increased coordinately (Fig. 2). With weak RNA-protein interactions, a given increment in $\beta$-galactosidase activity corresponded to a relatively large increase in 3-AT resistance; with strong interactions, the increment in 3-AT resistance was reduced. These data are consistent with the known requirement for tetramerization of $\beta$-galactosidase protein for enzyme activity (Cohn 1957). We conclude that HIS3 provides a more sensitive monitor of affinity with weaker RNA-protein interactions.

\section{Affinity versus reporter gene expression}

To determine the relationship between reporter gene activation and the strength of RNA-protein interactions, we first analyzed the affinity of purified recombinant FBF-1 for the same six RNAs. FBF-1 was generated by cleavage of a GST-TEV-FBF-1 protein with TEV protease. The purified protein was incubated with 28 -nt RNAs that had been $5^{\prime}$ end-labeled. A representative analysis, using FBEa-wt, is

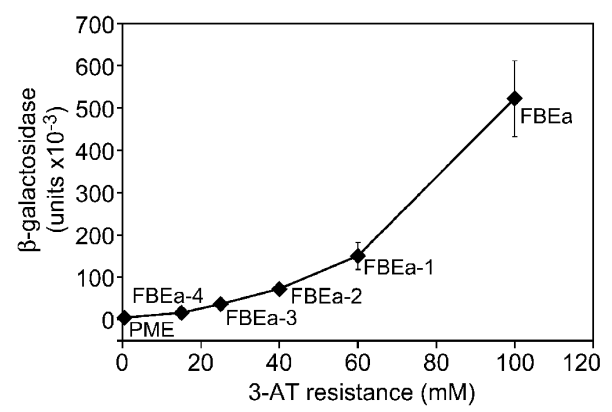

FIGURE 2. Comparison of HIS3 and LacZ expression. 3-AT resistance was quantified by plating cells on different concentrations of 3-AT. The highest concentration of 3-AT on which cells grew is plotted on the $\mathrm{X}$-axis. The $\beta$-galactosidase activity was measured using the Beta-Glo assay (see Materials and Methods). The light emission, in arbitrary units, is depicted on the Y-axis. Each point derived from analysis of a different RNA as indicated in the figure. 
shown in Figure 3A. The apparent $\mathrm{Kd}$ of this interaction, the strongest analyzed, was $\sim 10 \mathrm{nM}$.

For each RNA, Kd was compared to both HIS3 and $\beta$-galactosidase levels in the three-hybrid assay (Fig. 3B,C). The RNA sequences analyzed in the three-hybrid assay were the same as those used in vitro, except for requisite vector sequences. Kd's from 10 to $80 \mathrm{nM}$ were directly related to the $\log$ of $\beta$-galactosidase activity. In this range of $\mathrm{Kd}$, small changes result in substantial differences in enzyme activity, and $\beta$-galactosidase activity and 3-AT resistance were sensitive and reliable reflections of affinity. Even at the highest Kd examined ( $80 \mathrm{nM}), \beta$-galactosidase activity was substantially above background.

\section{Concentration of protein versus reporter expression}

To test the effects of intracellular protein concentration on reporter gene expression, a series of plasmids was used to express identical FBF-1/AD proteins at different intracellular concentrations. These plasmids vary in the strength of the promoters directing FBF-1/AD transcription, and in copy number (see Materials and Methods). Five plasmids, each carrying one of RNA, were introduced into each of the FBF-1/AD-producing strains. LacZ expression was quantified and displayed as a function of protein concentration (Fig. 4A). FBF-1/AD protein levels were determined by Western blotting, and varied over a 100 -fold range (Fig. $4 \mathrm{~B})$.

LacZ expression and protein concentration were directly
A

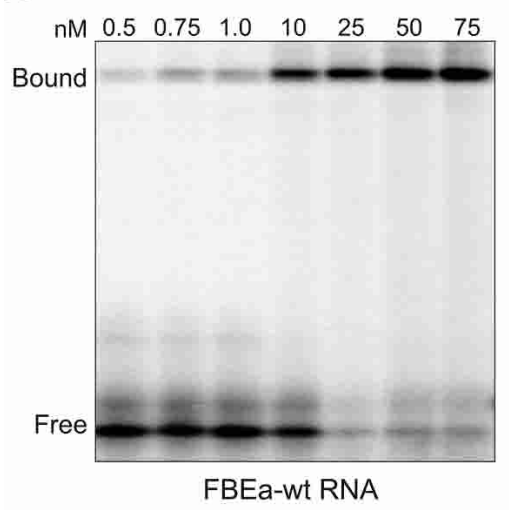

B

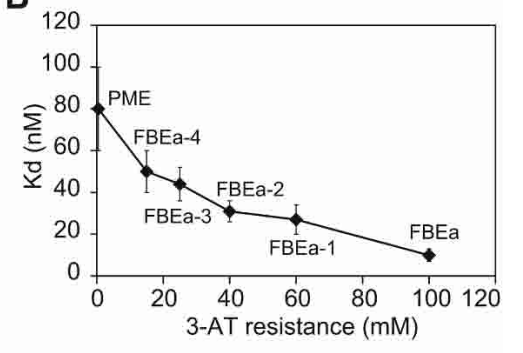

C

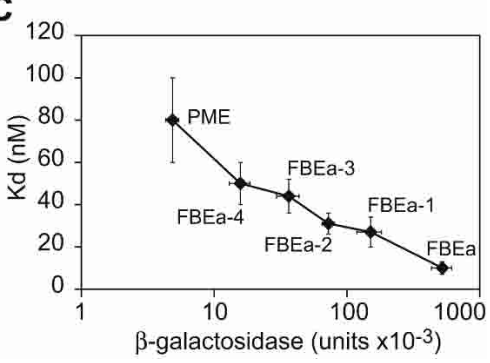

FIGURE 3. Comparison of in vitro binding affinities to reporter gene activation. $(A)$ Electrophoretic mobility shift assay with purified FBF-1 and $100 \mathrm{fmol}$ of $5^{\prime}$-end-labeled synthetic FBEa-wt RNA. Bound and free FBEa-wt RNA are labeled. The total concentration of FBF-1 present in each incubation is indicated above each lane. Analogous experiments were performed with each of the RNAs. (B) Kd versus 3-AT resistance. $(C) \mathrm{Kd}$ versus $\beta$-galactosidase activity.

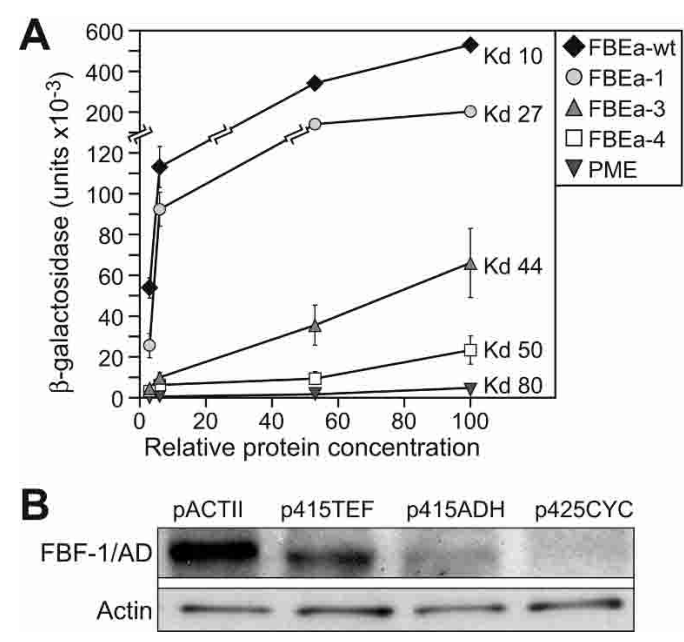

FIGURE 4. Effect of $\mathrm{AD}$ protein abundance on reporter expression. (A) Protein abundance versus LacZ activity. FBF-1/AD was produced at different levels in yeast using four different vectors. Y-axis: $\beta$-galactosidase activity. $\mathrm{X}$-axis: relative protein concentration, determined by Western blotting, and normalized to that of using the pACTII vector. $(B)$ Levels of FBF-1/AD determined by Western blotting using and anti-AD antibody. Anti-actin is used as a loading control.

related over a substantial range of Kd (Fig. 4A). High-affinity interactions could be detected using even low levels of FBF-1/AD, while weak interactions required high levels. However, even weak interactions, such as that between FBF-1/AD and the PME, yielded $\beta$-galactosidase levels significantly above background, and increased with protein concentration. Similarly, LacZ expression varies in concert with the affinity of the interaction. For example, for any given level of $\mathrm{FBF}-1 / \mathrm{AD}$ protein, the rank orders of RNA affinities and LacZ expression levels are the same (Fig. 4A).

\section{An improved yeast strain for library screening}

One common application of the threehybrid system concerns the identification of proteins that bind an RNA sequence of interest. In screens for proteins that bind to a specific RNA, the RNA is tethered to the promoter via a chimeric LexA/MS2 protein, and a library of DNAs encoding proteins and protein fragments is introduced by transformation. Colonies are obtained in which the HIS3 gene is activated, which enables growth in selective media. A common source of background in such screens is the interaction of polypeptides in the library with the protein bound to DNA. These so-called RNA- 
independent positives can constitute $>95 \%$ of the total number of colonies obtained. We sought to develop a strain that would mitigate this problem.

The common strains used in the three-hybrid system, such as L40coat, contain a fusion protein comprising a DNA-binding protein fused to MS2 coat protein (e.g., LexA/MS2). Coat protein must dimerize to bind its RNA ligand (Fig. 5A; Bardwell and Wickens 1990; Valegard et al. 1994; LeCuyer et al. 1995). We reasoned that monomers of MS2 coat protein tethered to the promoter would have an exposed surface that might bind proteins and protein fragments with high affinity, thereby activating the gene without binding the RNA. Moreover, monomers would be inactive in the assay and occupy LexA operators unproductively. We therefore created a new strain, YBZ1, carrying a tandem, head-to-tail dimer of MS2 coat protein fused to a single LexA monomer (Fig 5B; Peabody and Lim 1996). In the crystal structure of an MS2 coat protein dimer, the N and C termini are relatively close to one another (Valegard et al. 1994); a nine-amino-acid linker was introduced to enable flexibility in orienting the two MS2 segments. In addition, each coat protein segment also carries a point mutation $(\mathrm{N} 55 \mathrm{~K})$ that decreases the $\mathrm{Kd}$ of the RNA-protein

A. L40coat

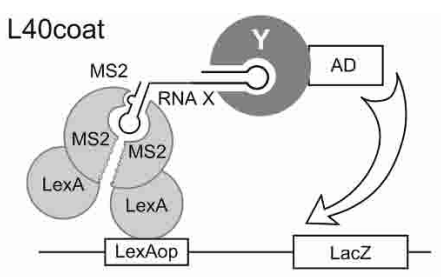

B. YBZ1

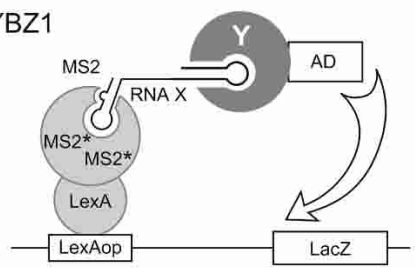

\begin{tabular}{|c|c|c|c|}
\hline C & Steps & L40coat & YBZ1 \\
\hline \multirow{3}{*}{1} & Transformants & $1,630,000$ & 800,000 \\
\hline & HIS $3+$ colonies & -3000 & 49 \\
\hline & $\begin{array}{r}\text { White colonies } \\
(A D E 2+)\end{array}$ & 259 & 33 \\
\hline 2 & $\beta$-gal positive & 60 & 9 \\
\hline & RNA-dependent & 29 & 8 \\
\hline \multicolumn{2}{|c|}{4 Sequence-specific } & 2 & 7 \\
\hline 5 & FBF & 0 & 3 \\
\hline
\end{tabular}

FIGURE 5. cDNA/AD library screen using YBZ1 and L40coat yeast strains. $(A)$ In the yeast strain L40coat, LexA is fused to a monomer of MS2 coat protein. (B) In strain YBZ1, a head-to-tail dimer of LexAMS2 coat protein fusion is introduced. MS2*, the MS2 coat protein used in YBZ1, possesses mutation N55K, which decreases $\mathrm{K}_{\mathrm{d}}$ sevenfold (Lim et al. 1994). (C) Three-hybrid screen in YBZ1 versus L40coat. Transformants were plated on $0.5 \mathrm{mM} 3$-AT to select for activation of HIS3. The identity of the RNA-dependent positives were determined by sequencing with pACT-specific primers. interaction sevenfold, from $3 \times 10^{-9}$ to $2 \times 10^{-10} \mathrm{M}$ (Lim et al. 1994).

We first compared reporter gene expression in YBZ1 and the closely related strain, L40coat, which carries a LexA/ MS2 monomer. We assayed LacZ activity in strains carrying FBF-1/AD in combination with eight RNAs that have varying affinities for FBF-1. The magnitude of signal is reproducibly $15 \%-30 \%$ higher in YBZ1.

To compare YBZ1 and L40coat in a genuine cDNA library screen, we used FBEa-wt as RNA "bait," and screened a C. elegans cDNA/AD library in both strains (Fig. 5C). FBF-1 and FBF-2 have previously been shown to bind the FBEa-wt and thereby regulate gld-1 mRNA in the C. elegans germ line (Crittenden et al. 2003). In the first step, $\sim 1$ million transformants were obtained in each strain. YBZ1 yielded roughly $5 \%$ the number of HIS3-positive transformants obtained with L40coat. To help identify the desired colonies, the RNA plasmid carried URA3 and ADE2 markers. Cells that activated HIS3 expression in the absence of the RNA plasmid could lose it; because the strains are ade2deficient, such colonies turned red, or red-sectored, and could be discarded. The fraction of the total number of transformants that were $\mathrm{ADE}^{+}$(white) was eightfold higher in YBZ1 than in L40 coat (70\% vs. 9\%). The $\beta$-galactosidase activity of these colonies was determined (Step 2): the two strains were comparable. Next, RNA plasmids were counterselected using 5-FOA, to identify with certainty those colonies that required the RNA plasmid to activate HIS3 ("RNA-dependent positives"; Step 3). Again, YBZ1 yielded a higher fraction of RNA-dependent colonies than did L40coat (89\% [8/9] vs. 48\% [29/60]). Finally, plasmids were extracted from yeast, transformed into Escherichia coli, and retested by transformation into naive yeast carrying either the specific RNA (FBEa-wt) or a control RNA (Step 4). Seven out of eight of the RNA-dependent positives obtained in YBZ1, and only two of the 29 obtained in L40coat, were specific for FBEa. To confirm these data, the identity of all of the RNA-dependent positives ( 29 in L40coat and eight in YBZ1) was determined by sequencing (Step 5). Three of the seven sequence-specific positives obtained in YBZ1 were FBF, while no FBF clones were obtained in the L40coat screen. Thus, a high fraction of the $\mathrm{HIS}^{+}{ }^{+} \mathrm{LacZ}^{+}$white colonies proved to be sequence-specific.

We conclude that the new strain yields a higher fraction of genuine, sequence-specific positives. It simplifies screening by drastically reducing the number of RNA-independent positives obtained in the initial transformation, while increasing the fraction of positives that are sequence-specific at multiple steps.

\section{DISCUSSION}

Our experiments permit three main conclusions. First, 3-AT resistance and $\beta$-galactosidase levels are directly related to Kd. Second, the phenotypic effects of variations in 
$\mathrm{Kd}$ are a function of the abundance of the AD fusion protein. Third, a new yeast strain, YBZ1, reduces background and increases the number of genuine positives obtained in a three-hybrid screen of cDNA/AD libraries.

For the RNA-binding protein FBF, Kd can be directly extrapolated from 3-AT resistance and $\beta$-galactosidase levels over at least a 10 -fold range of $\mathrm{Kd}$. That range is at least 10 to $100 \mathrm{nM}$. While the apparent dynamic range is limited, it likely can be extended by varying the $\mathrm{AD}$ vector and reporter genes, as documented for the two-hybrid system (Estojak et al. 1995). For example, higher levels of expression of the $\mathrm{AD}$ fusion protein should enhance detection of interactions weaker than $100 \mathrm{nM}$, while lower levels of the $\mathrm{AD}$ fusion protein will enable affinities to be extrapolated even in the subnanomolar range. Similarly, different proteins may vary quantitatively in the range of concentrations over which Kd can be extrapolated from phenotypes.

A different three-component system has been described to detect the interaction of small molecules with proteins (Licitra and Liu 1996). One permutation exploits a bifunctional ligand with a methotrexate (MTX) moiety (Fig. 6A). The ligand is anchored to the promoter by binding dihydrofolate reductase (DHFR), with a Kd in the low picomolar range. Binding of the other end of the ligand to a protein partner, Y, activates expression of the reporter gene. For example, an MTX-SLF (small molecule synthetic ligand) activates the reporter genes in the presence of a FK506binding protein 12 (FKBP12)/AD fusion protein (Drees 1999). In this system, the LexA/DHFR-MTX pair is analogous to the LexA/MS2-RNA pair used to assay RNA-protein interactions: similarly ligand $\mathrm{X}$ and RNA $\mathrm{X}$ are analogous (Fig. 6B).

The two systems display a remarkably similar dependence of LacZ expression on Kd. In a recent study, mutants of FKBP12/AD with a range of affinities were tested in combination with MTX-SLF ligands (de Felipe et al. 2004). In Figure 6C, we compare those data with our own, in which the ligand is RNA. The two sets of data are very similar. We infer that the two tethering interactions, MS2 coat protein with RNA and DHFR with MTX, are equivalent in the assays, although the difference in their Kd's is substantial $\left(10^{-10}\right.$ [MS2/RNA] vs. $10^{-14}$ [DHFR/MTX]). An implication of this finding is that further increases in the affinity of the protein/RNA tether will not significantly increase reporter gene expression in the RNA-based three-hybrid assay. Indeed, the mutation of MS2 coat protein present in YBZ1 enhances Kd $\sim 10$-fold yet has only a modest affect on LacZ expression levels.

One common application of the three-hybrid system has been to identify proteins in a CDNA/AD library that bind to an RNA sequence of interest. Earlier strains, including L40coat, have been used successfully for this purpose, with a wide variety of RNA/protein combinations (Bernstein et al. 2002). The background of "RNA-independent" positives

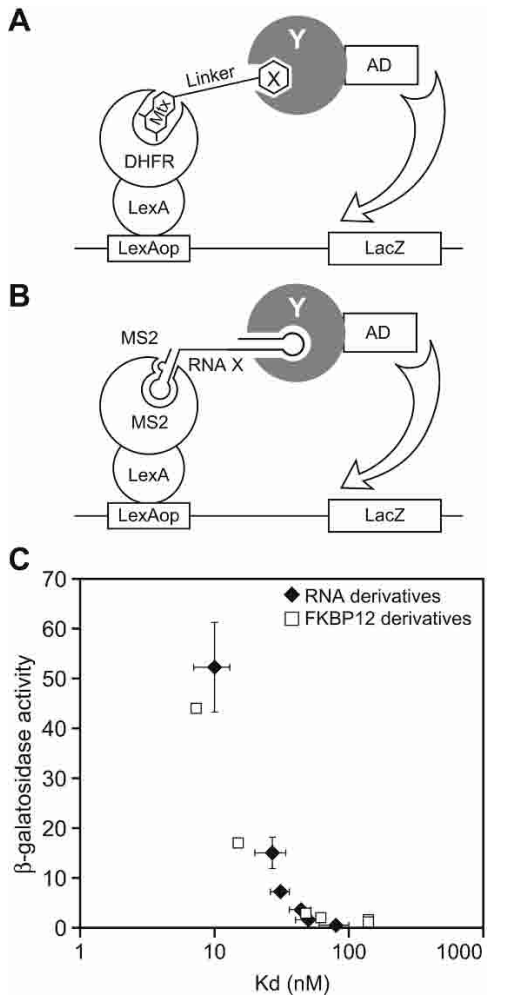

FIGURE 6. Comparison of three-hybrid systems to detect RNA and small molecule ligands with protein partners. $(A)$ The small-moleculebased three-hybrid system. Fusion proteins contain the LexA DNAbinding domain linked to dihyrdrofolate reductase, and protein $\mathrm{Y}$ with AD. A small molecule containing methotrexate linked to moiety $\mathrm{X}$ connects the two proteins and thereby leads to activation of the reporter gene. (B) RNA-based three-hybrid system. The RNA contains two MS2 stem loops fused to RNA sequence X. (C) Comparison of the relationship between $\mathrm{Kd}$ and reporter gene activity in the two systems. Data for the RNA-based system (Fig. 3C) are compared with the data of de Felipe et al. (2004).

in such screens can be high, and is a function of the level of HIS3 expression demanded. The high affinity of certain interactions can permit selection that demands high levels of HIS3 expression; in these cases, the fraction of all positives that prove to be genuine is high, even in strain L40coat. For example, the high affinity of the protein that binds the stem-loop at the end of histone mRNA has permitted it to be identified in three-hybrid screens with very little background (Wang et al. 1996; Martin et al. 1997). More often, however, the strength of the interaction is unknown; in these instances, it would be ideal to permit the detection of even weak interactions.

A new strain, YBZ1, facilitates three-hybrid screening of cDNA/AD libraries in multiple ways, including a dramatic reduction of background, RNA-independent positives. In directed tests of known combinations of RNA and protein, YBZ1 yields $15 \%-30 \%$ more $\beta$-galactosidase activity. This presumably reflects the presence of a point mutation in coat protein that enhances RNA binding. The new strain also carries LexA linked to an intramolecular dimer of MS2 coat 
protein, rather than a monomer as in past strains. The presence of the dimer appears to have at least four salutary effects in three-hybrid screens, related to the elimination of a promiscuous interacting surface in MS2 coat protein monomers. First, the dimer reduces the number of RNAindependent positives obtained in the initial transformation. LexA/MS2 monomer presumably presents a surface that can interact not only with another MS2 polypeptide, but with proteins and protein fragments encoded by the library. These yield colonies in the initial transformation that are of no interest and that do not appear with the dimer-containing strain. A second benefit of the LexA/MS2 coat protein dimer appears to be an enhancement of the fraction of RNA-dependent positives that are sequence-specific. This finding implies that, in the monomer-containing strains, some positives that require RNA also recognize the MS2 monomer's exposed surface. Third, the dimer appears to reduce an intrinsic background obtained with empty vectors in the monomer-containing strain. Endogenous yeast proteins may activate HIS3 expression by interacting with the exposed MS2 protein monomer. In the dimer-containing strain, these interactions disappear. Finally, the new strain yields more genuine positives (i.e., FBF in Fig. 5C) than does the strain carrying the LexA/MS2 monomer. This effect is more paradoxical: the presence of the dimer not merely decreases the background, but also increases the number of positives obtained. We infer that "nonspecific" interactions with the monomer surfaces may actually interfere with the binding of genuine positives. From a pragmatic perspective, the new strain clearly simplifies screening. It yields fewer initial transformants to analyze, and a higher fraction and absolute number of genuine, sequencespecific positives.

\section{MATERIALS AND METHODS}

\section{Plasmids for three-hybrid system}

\section{Activation domain plasmids}

The FBF-1 RNA-binding region (amino acids 121-614) was cloned into pACTII, an activation domain vector (Kraemer et al. 1999). To produce FBF-1 at different levels, the same AD-FBF-1 was cloned into p415TEF, 415ADH, and p425CYC1 (Mumberg et al. 1995).

\section{RNA plasmids}

The RNA sequences of FBEa and PME were cloned into pIIIAMS2-2, as described previously (Zhang et al. 1997; Crittenden et al. 2003).

\section{Yeast three-hybrid tests and screens}

In all experiments, RNA plasmids were cotransformed with FBF-1 into YBZ1 or L40coat yeast strains.
The genotype of YBZ1 is MATa, ura3-52, leu2-3, 112, his3-200, trp1-1, ade2, LYS2 :: (LexAop)-HIS3, ura3 :: (lexA-op)-lacZ, LexAMS2 coat (N55K). The two coat protein monomers were joined by a peptide linker of Gly-Ala-Pro-Gly-Ile-His-Pro-Gly-Met.

The three-hybrid screen described in Figure 5C was performed as described in Bernstein et al. (2002), and detailed in the legend to Figure $5 \mathrm{C}$.

\section{Electrophoretic mobility shift assays and FBF-1 protein}

The approximate dissociation constants of FBF-1 binding to various RNAs were determined by electrophoretic gel mobility shift assay. RNAs were prepared by chemical synthesis (Dharmacon) and $5^{\prime}$-end-labeled. The concentration of RNA in all assays was $100 \mathrm{pM}$. Buffer conditions for binding were $10 \mathrm{mM}$ HEPES ( $\mathrm{pH}$ 8.0), $1 \mathrm{mM}$ EDTA, $50 \mathrm{mM} \mathrm{KCl}, 2 \mathrm{mM}$ DTT, $0.1 \mathrm{mg} / \mathrm{mL}$ BSA, $0.02 \%$ Tween-20, and $0.2 \mathrm{mg} / \mathrm{mL}$ tRNA. Incubations were performed for $30 \mathrm{~min}$ at room temperature. To each $10 \mu \mathrm{L}$ of incubation, $2.5 \mu \mathrm{L}$ of loading dye $(30 \%[\mathrm{v} / \mathrm{v}]$ glycerol, $0.05 \%[\mathrm{w} / \mathrm{v}]$ xylene cyanol) was added. Samples were analyzed on a pre-run native polyacrylamide gel $(6 \%$ [w/v] 29:1 acrylamide/Bis-acrylamide, $0.5 \times \mathrm{TBE}$ ). Gels were resolved at $200 \mathrm{~V}$ at $4^{\circ} \mathrm{C}$ for $4 \mathrm{~h}$. The fraction of bound and unbound RNA was determined using ImageQuant (Amersham). Kd, defined as the concentration of protein at which half-maximal binding occurs, was determined using GraphPad Prism 4.

FBF-1 was prepared from $E$. coli carrying the plasmid pGex6P-1. In this plasmid, FBF-1 (amino acids 121-614) is preceded by a TEV protease cleavage site at its $\mathrm{N}$ terminus. Protein concentration was determined using the Bradford assay and corroborated using SDS-PAGE. Details of FBF-1 purification will be published elsewhere (D. Bernstein, B. Hook, and M. Wickens, in prep.).

\section{$\beta$-Galactosidase activity measurements}

To quantify the level of LacZ expression, we used a reagent developed by Promega, which they refer to as the Beta-Glo Assay System. The substrate is cleaved by $\beta$-galactosidase to liberate luciferin, which then is acted on by luciferase to generate light. The reagent provided also lyses cells, enabling rapid assays. The assay was developed for mammalian cells. We performed a series of experiments to determine whether it was suitable for Saccharomyces cerevisiae, and to optimize the assay for use in the three-hybrid system.

To optimize the assay, we used a strain carrying FBF-1/AD and RNA-MS2 fusions having a range of affinities and magnitudes of reporter gene expression. We determined that:

1. The optimum time of incubation with the Beta-Glo reagent is $1 \mathrm{~h}$.

2. The assay is linear over a 10,000-fold range of yeast density. A yeast culture with an optical density of 1.0 was serially diluted, and $\beta$-galactosidase was assayed under standard conditions.

3. Yeast in exponential growth $\left(0.1-0.8 \mathrm{~A}_{600}\right)$ versus stationary phase $\left(>1.0 \mathrm{~A}_{600}\right)$ were very similar in relative activity. However, exponentially growing cells reproducibly yielded $25 \%-$ $30 \%$ more activity.

4. Freezing the yeast prior to the assay decreases the signal.

5. Yeast are killed by $1 \mathrm{~h}$ of incubation with the reagent. 
6. The standard assay protocol follows. Yeast cultures were grown in selective media to log phase $(0.1-0.8)$. Cultures were diluted to an $\mathrm{A}_{600}$ of 0.1. An equal volume of Beta-Glo reagent was then added, and the mixture was incubated at room temperature for $60 \mathrm{~min}$. The sample was then read in a luminometer (Monolight 2010).

In the figures, results are presented as " $\beta$-galactosidase (units $\times$ $10^{-3}$ )" activities were normalized to empty vector. The units are arbitrary light units recorded by the luminometer.

\section{ACKNOWLEDGMENTS}

We thank Christian Eckmann for plasmids expressing FBF at different levels, and members of the Wickens laboratory for helpful discussions. This work was supported by NIH research grants to M.W.

Received October 11, 2004; accepted November 17, 2004.

\section{REFERENCES}

Bardwell, V. and Wickens, M. 1990. Purification of RNA and RNAprotein complexes by R17coat protein affinity chromatography. Nucleic Acids Res. 18: 6587-6594.

Bernstein, D.S., Buter, N., Stumpf, C., and Wickens, M. 2002. Analyzing mRNA-protein complexes using a yeast three-hybrid system. Methods 26: 123-141.

Celander, D.W., Bennett, K.A., Fouts, D.E., Seitz, E.A., and True, H.L. 2000. RNA challenge phages as genetic tools for study of RNAligand interactions. Methods Enzymol. 318: 332-350.

Cohn, M. 1957. Contributions of studies on the $\beta$-galactosidase of Escherichia coli to our understanding of enzyme synthesis. Bacteriol. Rev. 21: 140-168.

Crittenden, S.L., Bernstein, D.S., Bachorik, J.L., Thompson, B.E., Gallegos, M., Petcherski, A.G., Moulder, G., Barstead, R., Wickens, M., and Kimble, J. 2002. A conserved RNA-binding protein controls germline stem cells in Caenorhabditis elegans. Nature 417: 660-663.

Crittenden, S.L., Eckmann, C.R., Wang, L., Bernstein, D.S., Wickens, M., and Kimble, J. 2003. Regulation of the mitosis/meiosis decision in the Caenorhabditis elegans germline. Philos. Trans. $R$ Soc. Lond. B Biol. Sci. 358: 1359-1362.

Cullen, B.R. 2000. Principles and applications of a Tat-based assay for analyzing specific RNA-protein interactions in mammalian cells. Methods Enzymol. 328: 322-329.

de Felipe, K.S., Carter, B.T., Althoff, E.A., and Cornish, V.W. 2004. Correlation between ligand-receptor affinity and the transcription readout in a yeast three-hybrid system. Biochemistry 43: 10353-10363.

Drees, B.L. 1999. Progress and variations in two-hybrid and threehybrid technologies. Curr. Opin. Chem. Biol. 3: 64-70.

Estojak, J., Brent, R., and Golemis, E.A. 1995. Correlation of twohybrid affinity data with in vitro measurements. Mol. Cell. Biol. 15: 5820-5829.

Harada, K., Martin, S.S., and Frankel, A.D. 1996. Selection of RNAbinding peptides in vivo. Nature 380: 175-179.

Harada, K., Horiya, S., Zehavi, H., and Frankel, A.D. 2000. Screening in vivo for RNA-binding peptides from combinatorial libraries. Nucleic Acids Symp. Ser. 269-270.

Jain, C. and Belasco, J.G. 1996. A structural model for the HIV-1 Rev-RRE complex deduced from altered-specificity rev variants isolated by a rapid genetic strategy. Cell 87: 115-125.

- 2000. Rapid genetic analysis of RNA-protein interactions by translational repression in Escherichia coli. Methods Enzymol. 318: 309-332.
Kollmus, H. and Hauser, H. 2000. Frameshifting assay to characterize RNA-protein interactions in eukaryotic cells. Methods Enzymol. 318: 363-374.

Kraemer, B., Crittenden, S., Gallegos, M., Moulder, G., Barstead, R., Kimble, J., and Wickens, M. 1999. NANOS-3 and FBF proteins physically interact to control the sperm-oocyte switch in Caenorhabditis elegans. Curr. Biol. 9: 1009-1018.

Laird-Offringa, I.A. 1999. In vitro genetic analysis of RNA-binding proteins using phage display. Methods Mol. Biol. 118: 189-216.

Landt, S.G., Tan, R., and Frankel, A.D. 2000. Screening RNA-binding libraries using Tat-fusion system in mammalian cells. Methods Enzymol. 318: 350-363.

LeCuyer, K.A., Behlen, L.S., Uhlenbeck, O.C. 1995. Mutants of the bacteriophage MS2 coat protein that alter its cooperative binding to RNA. Biochemistry 34: 10600-10606.

Licitra, E.J. and Liu, J.O. 1996. A three-hybrid system for detecting small ligand-protein receptor interactions. Proc. Natl. Acad. Sci. 93: $12817-12821$.

Lim, F., Spingola, M., and Peabody, D.S. 1994. Altering the RNA binding specificity of a translational repressor. J. Biol. Chem. 269: 9006-9010.

Martin, F., Schaller, A., Eglite, S., Schumperli, D., and Muller, B. 1997. The gene for histone RNA hairpin binding protein is located on human chromosome 4 and encodes a novel type of RNA binding protein. EMBO J. 16: 769-778.

Mumberg, D., Muller, R., and Funk, M. 1995. Yeast vectors for the controlled expression of heterologous proteins in different genetic backgrounds. Gene 156: 119-122.

Paraskeva, E. and Hentze, M.W. 2000. Translational repression assay procedure: A method to study RNA-protein interactions in yeast. Methods Enzymol. 318: 374-384.

Park, Y.W., Tan, S.L., and Katze, M.G. 1999. Differential sensitivity to 5-fluoro-orotic acid as a screen for bait RNA-independent false positives in a yeast three-hybrid system. Biotechniques 26: 11021106.

Peabody, D.S. and Lim, F. 1996. Complementation of RNA binding site mutations in MS2 coat protein heterodimers. Nucleic Acids Res. 24: 2352-2359.

Peled-Zehavi, H., Smith, C.A., Harada, K., and Frankel, A.D. 2000. Screening RNA-binding libraries by transcriptional antitermination in bacteria. Methods Enzymol. 318: 297-308.

SenGupta, D.J., Zhang, B., Kraemer, B., Pochart, P., Fields, S., and Wickens, M. 1996. A three-hybrid system to detect RNA-protein interactions in vivo. Proc. Natl. Acad. Sci. 93: 8496-8501.

SenGupta, D.J., Wickens, M., and Fields, S. 1999. Identification of RNAs that bind to a specific protein using the yeast three-hybrid system. RNA 5: 596-601.

Valegard, K., Murray, J.B., Stockley, P.G., Stonehouse, N.J., and Liljas, L. 1994. Crystal structure of an RNA bacteriophage coat proteinoperator complex. Nature 371: 623-626.

Wang, Z.F., Whitfield, M.L., Ingledue III, T.C., Dominski, Z., and Marzluff, W.F. 1996. The protein that binds the $3^{\prime}$ end of histone mRNA: A novel RNA-binding protein required for histone premRNA processing. Genes \& Dev. 10: 3028-3040.

Wickens, M., Bernstein, D., Crittenden, S., Luitjens, C., and Kimble, J. 2001. PUF proteins and 3'UTR regulation in the Caenorhabditis elegans germ line. Cold Spring Harb. Symp. Quant. Biol. 66: 337343.

Wickens, M., Bernstein, D.S., Kimble, J., and Parker, R. 2002. A PUF family portrait: 3'UTR regulation as a way of life. Trends Genet. 18: $150-157$.

Xie, B., Calabro, V., Wainberg, M.A., and Frankel, A.D. 2004. Selection of TAR RNA-binding chameleon peptides by using a retroviral replication system. J. Virol. 78: 1456-1463.

Zhang, B., Gallegos, M., Puoti, A., Durkin, E., Fields, S., Kimble, J., and Wickens, M.P. 1997. A conserved RNA-binding protein that regulates sexual fates in the C. elegans hermaphrodite germ line. Nature 390: $477-484$. 

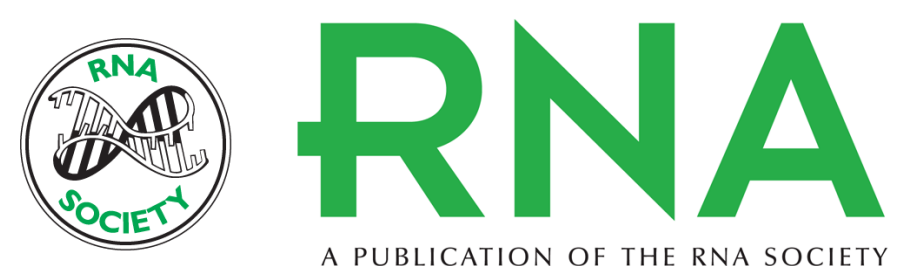

A PUBLICATION OF THE RNA SOCIETY

\section{RNA-protein interactions in the yeast three-hybrid system: Affinity, sensitivity, and enhanced library screening}

BRAD HOOK, DAVID BERNSTEIN, BEILIN ZHANG, et al.

RNA 2005 11: 227-233

References This article cites 33 articles, 8 of which can be accessed free at: http://rnajournal.cshlp.org/content/11/2/227.full.html\#ref-list-1

License

Email Alerting Receive free email alerts when new articles cite this article - sign up in the box at the Service top right corner of the article or click here. 